\title{
A new variation of tracheobronchoplastic operation
}

\author{
A new variation of tracheobronchoplastic operations that we named modified plastic surgery was \\ applied successfully on six patients with lung cancer and one with carcinoid adenoma. Modified plastic \\ surgery consists of adaptive shaping of healthy portions of the bronchi and elimination of parts \\ affected by the pathologic process, which usually required the respective lung resection. Suturing of the \\ fragments reestablished the airways and rendered the formation of postoperative stenoses and anasto- \\ moses practically impossible. Indications for this type of operation were decided individually according \\ to the location of the process and the requirements for complete resection in the cases with lung \\ cancer. In many instances this type of operation was the only option for preservation of the organ. (J \\ ThORaC CaRdiovasc Surg 1994;107:29-31)
}

A. Simeonov, MD, PhD, Sofia, Bulgaria

I $n$ the field of thoracic surgery, there are three wellknown and routinely applied methods of tracheobronchoplasty. The so-called sleeve resections are most popular and involve end-to-end anastomoses. The indications for side-to-end anastomoses are more specific. An example is Grillo's ${ }^{1}$ operation which includes resection of the tracheal carina and end-to-end anastomosis of the right main bronchus to the trachea with subsequent anastomosis of the left main bronchus to the wall of the secondary bronchus. The third type of anastomosis is achieved by suturing side-to-side the walls of main, lobar, or segmental bronchi and anastomosis of the constructed double-barelled configurations to the lumen of the proximal tracheobronchial tree. In this manner, a new tracheal carina may be formed. We have had abundant practical experience with these routine plastic operations.

In August 1985 at the Thoracic Clinic of the National Center of Oncology, Sofia, Bulgaria, we performed a new modification of restoration of the tracheobronchial tree after right pneumonectomy and resection of the tracheobronchial carina. A 51-year-old male patient had lung cancer involving the posterior-inferior portion of the right main bronchus, the tracheal carina, and the proximal part

\footnotetext{
From the Thoracic Department of the National Center of Oncology, Sofia, Bulgaria.

Received for publication Feb. 18, 1993.

Accepted for publication April 20, 1993.

Address for reprints: A. Simeonov, MD, PhD, Associate Professor, 37 Vitosha Blvd., Sofia 1000, Bulgaria.

Copyright 1994 by Mosby-Year Book, Inc.

$0022-5223 / 94 \$ 1.00+.10 \quad 12 / 1 / 49680$
}

of the left main bronchus. In this case, the healthy cranial part of the right main bronchus was preserved and used as plastic material to cover the defect in the proximal part of the left main bronchus (Fig. 1, $A$ ). The success of this operation inspired us to work on the technique of this new type of tracheobronchial plastic surgery and the performance of different variations that we named modified plastic surgery.

\section{Patients and methods}

Modified plastic surgery consists of adaptive shaping of healthy portions of the bronchi and elimination of the parts affected by the pathologic process, which usually requires the respective pulmonary resection. The requirement has to be met for reestablishment of the patency of the airway after suturing the fragments. In all cases, patency was reestablished by means of monophylic nylon thread and Z-shaped sutures spaced 2 to $3 \mathrm{~mm}$ apart. These stages of operation in our patients were performed after manual preparation of the vessels for right upper lobectomy, lower bilobectomy, and right pneumonectomy.

Modified plastic surgery was performed on six patients with squamous cell carcinoma of the lung and one with bronchial adenoma (carcinoid type). In three of the patients with carcinoma, the disease was staged as T2 N1, and, in the other three, it was staged as T3 N0. For these patients, the following operations were performed: Four lower bilobectomies of two types (Fig. 1, $A$ and $B$ ), one upper lobectomy (Fig. 1, C), and one right pneumonectomy (Fig. 1, D). In all cases, the tumor had predominantly endobronchial distribution. In the patients with cancer who had partial lung resections, pneumonectomy was contraindicated because of functional reasons. Resection was complete as proved by frozen slides of the resection lines.

The patient with bronchial adenoma (Fig. 2) had a tumor facing the lumen of the left upper lobe bronchus. It was diagnosed after several episodes of pneumonia and massive hemoptysis. We performed only resection and modified bronchoplas- 


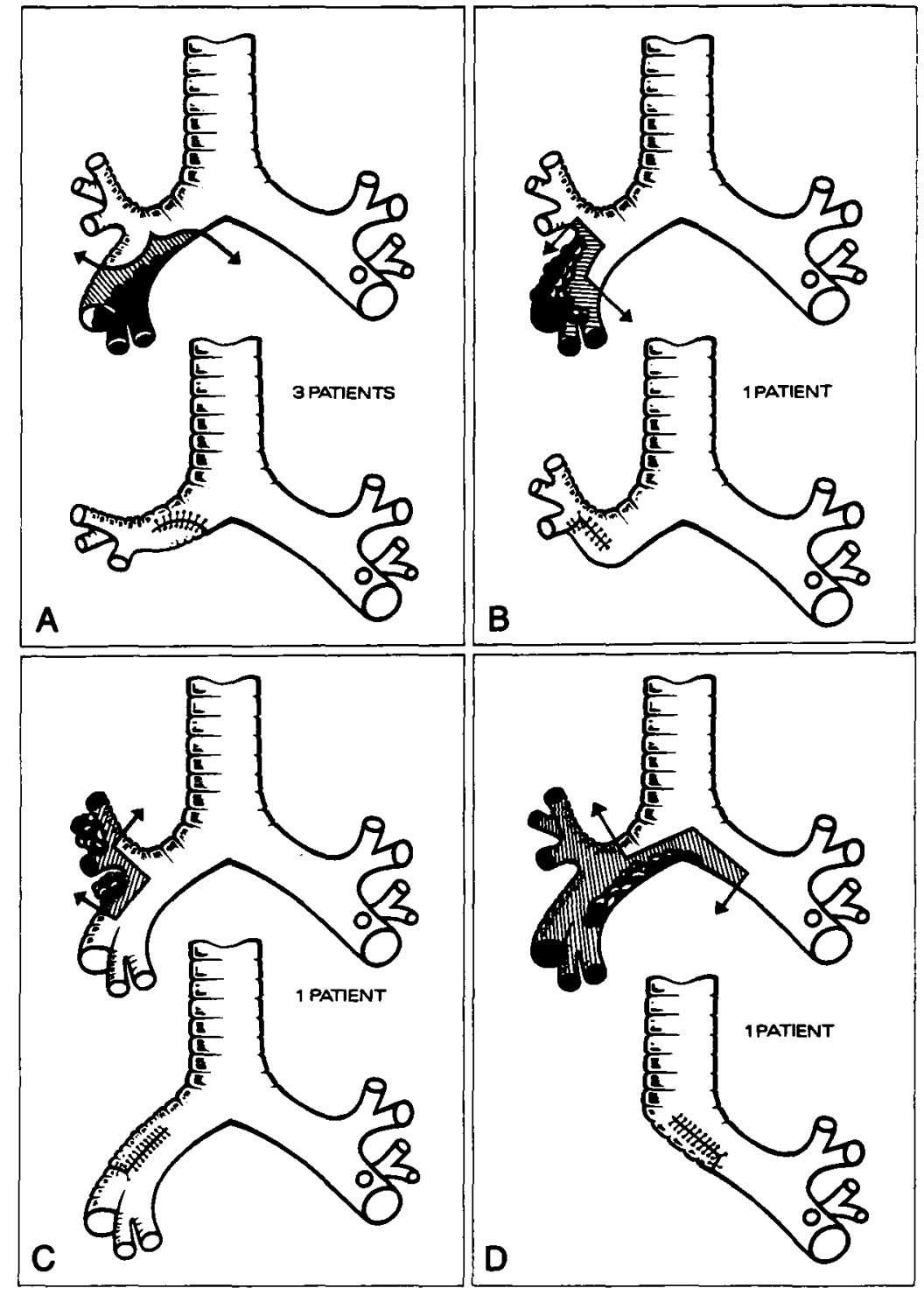

Fig. 1. A and B, Two types of lower bilobectomy with repair; C, upper lobectomy with repair; D, right pneumonectomy with repair.

ty, preserving the lung parenchyma. For good access to the bronchus, the pulmonary artery and inferior pulmonary vein were held aside.

\section{Results}

The postoperative period was uncomplicated in all seven patients. Control bronchoscopy on days 20 to 25 revealed good healing and the formation of a fine scar.

Two of the patients with lung cancer died of distant metastases 2 years after the operation: one with upper right lobectomy and the other with right pneumonectomy. The other patients are being followed-up. They were well at the time this article was written and had no signs of recurrence or distant metastases. The patient with bronchial adenoma recovered and was healthy.

\section{Discussion}

We did not find modified repair of the type that we are describing in the available literature. Only Hoshi and 


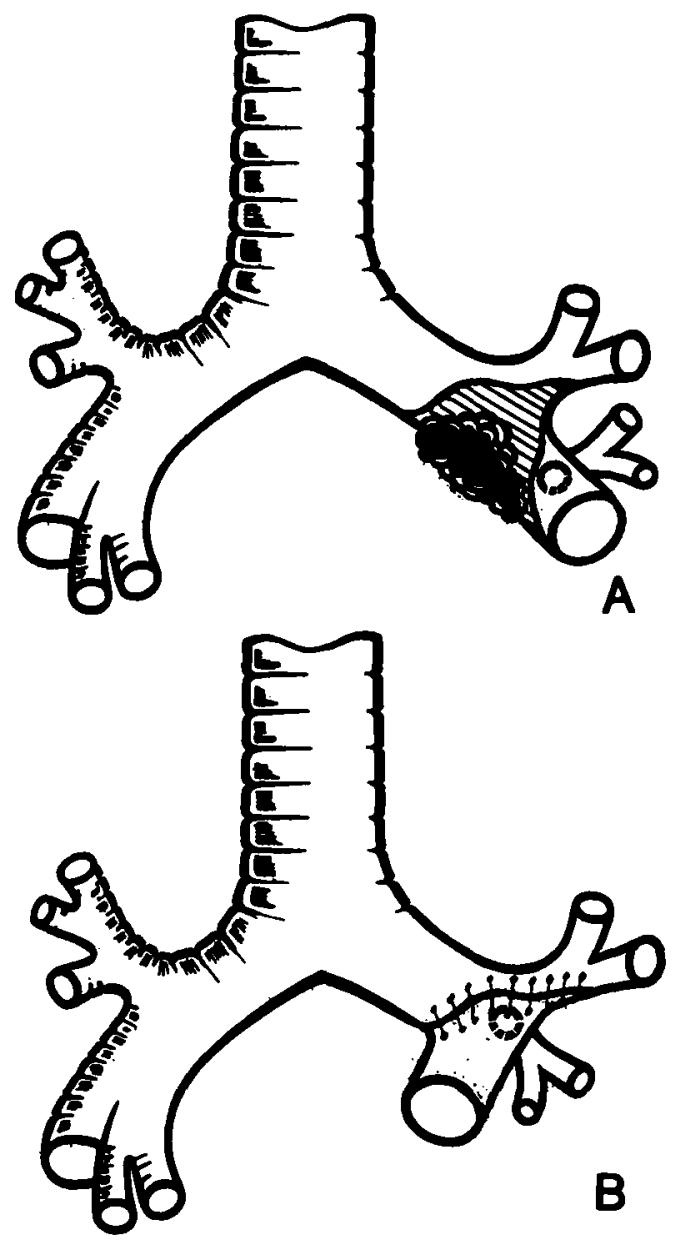

Fig. 2. Modified bronchoplasty with preservation of lung parenchyma.

associates $^{2}$ reported the correction of a defect of the tracheal carina after right pneumonectomy with a fragment of the right main bronchus. This work was done in 1990, and our first case was in August 1985.

This new variation of tracheobronchoplasty is relatively less frequently applicable in patients with lung cancer because of the requirement for completeness of resection. For instance, for the three patients (Fig. 1, $A$ ) who had indications for right lower bilobectomy, an alternative choice was resection of the right upper lobe bronchus and an end-to-end anastomosis with the main bronchus. Our experience with such cases shows that additional preparation of the main bronchial fragment is needed to reduce its lumen to match the dimensions of the right upper lobe bronchus. We have achieved this most frequently with wedge resection of the membranous part. In the other case (Fig. 1,B), because of involvement of the inferior aspect of the right upper lobe bronchus, there was no other choice for organ-preserving surgical procedures. There was a similar situation with the patient with right upper lobectomy (Fig. 1, C) because of engagement of part of the secondary bronchus by metastatic lymph nodes. The patient with right pneumonectomy would have had a practically inoperable condition if modified repair had not been applied. The patient with adenoma of the left bronchus would have had to have undergone lower lobectomy if anastomosis of the left upper lobe with the main bronchus had been the chosen procedure.

These considerations practically outline the indications for modified repair of the tracheobronchial tree. In this manner, they emerge as a new variation of organpreserving operations. The specific approach is decided for each case separately, with regard for the location of the pathologic process and the requirements for complete resection in the patients with lung cancer.

One advantage of this kind of modified plastic surgery over sleeve resection is that it renders the development of postoperative stenoses in the area of the anastomosis practically impossible, and the potential granulation formation does not lead to obstruction.

\section{REFERENCES}

1. Grillo HC. Management of cervical and mediastinal lesions of the trachea. JAMA 1966;197:1085-90.

2. Hoshi E, Kobayashi M, Aoyama T, et al. Two cases of wedge pneumonectomy in primary lung cancer involving the carina. Kyobu Geka 1990;43:226-30. 